\title{
Unix Version of CALOR89 for Calorimeter Applications ${ }^{1}$
}

ANL-HEP-TR--92-38

T. Handler

DE92 016175

University of Tennessee, Knoxville TN 37996

P.K. Job and L.E. Price

Argonne National Lab, Argonne IL 60439

T.A. Gabriel

Oak Ridge National Lab, Oak Ridge, TN 37831

\section{DISCLAIMER}

This report was prepared as an account of work sponsored by an agency of the United States Government. Neither the United States Government nor any agency thereof, nor any of their empioyees, makes any warranty, express or implied, or assumes any legal liability or responsibility for the accuracy, completeness, or usefulness of any information, apparatus, product, or process disclosed, or represents that its use would not infringe privately owned rights. Reference herein to any specific commercial product, process, or service by trade name, trademark, manufacturer, or otherwise does not necessarily constitute or imply its endorsement, recommendation, or favoring by the United States Government or any agency thereof. The views and opinions of authors expressed herein do not necessarily state or reflect those of the United States Government or any agency thereof.

\footnotetext{
${ }^{1}$ Work supported in part by US Department of Energy, Contract No. W-31-109-ENG-38, No. DE-AC05-84OR21400 and No. DE-AS05-ER03956

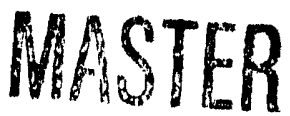

DISTRIBUTION OF THIS DOCUMENT IS UMLIm

The submittod manuccript has been authored by a contractor of the U.S. Govisnment under contract No. W.31.108ENG.38. Accordlngly, the U.S. Government rotwint : nonexclusive, royalty-ireo licons to pusslish or roprodure the publistied form of tivis contribution, or allow others to do $20, \mathrm{for}$ U.S. Government surnows.
} 


\section{SDC \\ SOLENOIDAL DETECTOR NOTES}

\section{Unix Version of CALOR89 for Calorimeter Applications}

T. Handler

Department of Physics and Astronomy

University of Tennessee, Knoxville, Tennessee 37966

P. K. Job, 'nd L. E. Price

High Energy Physics Division

Argonne National Laboratory, Argonne, Illinois 60439

T. A. Gabriel

Enginet,ing, Physics, and Mathematics Division

Oak Ridge National Laboratory, Oak Ridge, Tennessee 37831 


\section{Introduction}

CALOR89[1] is a system of coupled Morte Carlo particle transport computer codes (figure 1) which has been successfully employed for the estimation of calorimeter parameters in High Energy Physics [2][3]. In the past CALOR89 has been running on various IBM machines and on CRAY X-MP at Lawrence Livermore Lab. These machines had non-unix operating systems. In this report we present a UNIX version of CALOR89, which is especially suited for the UNIX work stations. Moreover CALOR89 is also been supplemented with two new program packages which makes it more user friendly. CALPREP is a program for the preparation of the input files for CALOR89 in general geometry[4] and ANALYZ is an analysis package to extract the final results from CALOR89 relevant to calorimeters. This report also provides two script files LCALOR and PCALOR. LCALOR runs CALOR89 sequence of programs and EGS4 for a given configuration sequentially on a single processor and PCALOR concurrently on a multiprocessor unix workstation.

\section{Program CALPREP}

CALOR89 consists of a number of independent programs like HETC[5], EGS4[6] and MORSE[7] working on the same problem. These programs were developed independently in the past for different applications. Therefore they have different input formats and specifications. This made the input preparation for CALOR89 very difficult and vulnerable to mistakes. It will be helpful to have a single input data file for a given run which controls the generation of all the input specifications for different programs in CALOR89. This will avoid inconsistent and redindant input to various programs. The legibility of the input data is also improved by providing a namelist input file.

CALPREP is a program which reads a single input file, CALPREP.INP consisting of information on incident particle and the configuration. In addition to this CALPREP also requires '*.MOR' and '*.HETC' files in the same directory, where '*' is the pegs media name. These are input media specifications for a given material 
for HETC and MORSE. These files for the elements and compounds commonly in use must be prepared only once like the pegs cross section file. CALPREP generates six output files which serve as input files to various CALOR89 programs. It also generates the geometry file, in general geometry format[4], which is read by all the CALOR89 programs. This saves the problem of preparing seven different input files into just one file. Figure 2 gives the schematic diagram of the CALPREP program. Appendix A ard B provides the listing of the CALPREP.INP and the .SCRIPT files. The input prameters are explained in detail in Appendix A. Table I gives the complete set of input and output files called by each of the CALOR89 programs. The '.bin' files are the cross section data bases. The output print files of all programs are denoted as '.ppr'.

\section{Program ANALYZ}

ANALYZ is a program to combine the results of various CALOR89 programs and EGS4. This reads a user provided input file and five output files from CALOR89 and EGS4. The output of ANALYZ is a print file called ANALYZ.OUT and a histogram file for PAW called ANALYZ.HST. ANALYZ provides information on several parameters of interest for the calorimeter designers. The package provides information on compensation characieristics and hadronic and electromagnetic resolutions as a function of shower integration time. It also gives the shower depth profiles for both the hadronic and electromagnetic showers. ANALYZ can be recoded for user specifis: applications. In the present form it gives a near complete set of information for a given configuration. Figure 3 gives the schematic diagram of the ANALYZ package. Appendix $\mathrm{C}$ provides information on ANALYZ.INP.

\section{Script Programs LCALOR and PCALOR}

LCALOR is a script program which It runs the entire sequence of CALOR89 program for hadrons and EGS4 for electrons for a given energy and configuration without the users intervention and analyses the results using the program ANALYZ. The input files generated by CALPREP and ANAIYYZINP must be in the 
same directory from which LCALOR is submitted. In LCALOR, the appropriate pathnames for the executable programs of CALOR89 (exec. ${ }^{*}$ ) must be provided once, depending upon the installation. For a given installation LCALOR need not be changed afterwards. LCALOR also requires a file called lightm.dat in the same directory which is the light curves for law energy neutrons read by MORSE. Figure 4 gives the schematic diatram of running CALDR89 and EGS4 using LCALOR. Appendix D gives a listing of LCALOR script program.

The sequence of CALOR89(fig.1) shows that once HETC88 is done for a given number of hadron events, SPECT89, EGS4 and MORSE can be run concurrently on the hadronic, electromagnetic and neutronic components of the hadron shower. EGS4 can also run concurrently for pure electromagnetic showers created by the incident electrons. With the advent of multiprocessor machines this became possible. PCALOR utilizes this property to speed up CALOR89. In this way on a multiprocessor workstation CALOR89 programs can be made to run concurrently as long as the HETC output file is on the disk. As in LCALOR the pathnames of the files in PCALOR must be changed once for a given installation. The input files including the geometry must be in the same directory as PCALOR. Figure 5 gives the schematic diagram of running CALOR89 using PCALOR. Appendix E gives the listing of the PCALOR script program.

\section{Summary}

In this report we have presented a unix version of CALOR89. The difficult problem of preparing several input files for the different programs of CALOR89 has been solved by CALPREP. An analysis program ANALYZ is included which gives many calorimeter parameters of interest. Two script programs are written for running CALOR89 sequentially on single processor and concurrently on multiprocessor unix workstations. 


\section{References}

[1] T.A. Gabriel et al.,"CALOR, A Monte carlo program package for the design and analy sis of the calorimeter systems", ORNL/TM 5619 (1977).

[2] T.Handler et al.,"CALOR89 Calorimeter simulations, Benchmarking and design calculations", Proc. Symp.on Detector Research and Development for SSC, FortWorth, Edited by T.Dombeck et al., World Scientific(1990)608.

[3] P.K. Job et al.,"Comparison of CALOR89 model predictions with scintillator plate calorimeter data", Nucl.Instr.and Meth., A309(1991)60.

[4] HET'C User Manual, KSIC code collection, ORNL-CCC-178C.

[5] T.W. Armstrong and K.C.Chandler, HETC- A High Energy Transport Code, Nucl.Sci.and Engg.49(1972)110.

[6] W.R. Nelson, H.Hirayama and D.W.O.Rogers, EGS4 Code System, SLAC 265(1985).

[7] M.B. Emmett and N.M. Greene, Morse Code System, ORNL/TM-3706 (1973). 
Table I

List of Input and Output Files of CALOR89 Programs

\begin{tabular}{|c|c|c|}
\hline Program Name & Input Files & Output Files \\
\hline $\begin{array}{l}\text { HETC } \\
\text { exec.hetc }\end{array}$ & $\begin{array}{l}\text { hetch.inp } \\
\text { *.geo } \\
\text { cascade.bin } \\
\text { evapor.bin }\end{array}$ & $\begin{array}{l}\text { *.dat } \\
\text { hetch.ppr }\end{array}$ \\
\hline $\begin{array}{l}\text { SPECT } \\
\text { exec.spect }\end{array}$ & $\begin{array}{l}\text { spect.inp } \\
\text { *.dat } \\
\text { kb.dat }\end{array}$ & $\begin{array}{l}\mathrm{s}^{*} . \mathrm{dat} \\
\text { spect.ppr }\end{array}$ \\
\hline $\begin{array}{l}\text { EGSPREPH } \\
\text { exec.fgsprep }\end{array}$ & $\begin{array}{l}\text { egspreph.inp } \\
* \text {.dat }\end{array}$ & $\begin{array}{l}\text { egspreph.out } \\
\text { egspreph.ppr }\end{array}$ \\
\hline $\begin{array}{l}\text { EGSH } \\
\text { exec.egs }\end{array}$ & $\begin{array}{l}\text { egsh.inp } \\
\text { egspreph.ovt } \\
\text { pegs.dat } \\
\text { *.geo }\end{array}$ & $\begin{array}{l}e^{*} \cdot \text { dat } \\
\text { egsh.ppr }\end{array}$ \\
\hline $\begin{array}{l}\text { MORSEH } \\
\text { exec.morse }\end{array}$ & $\begin{array}{l}\text { morseh.inp } \\
\text { *.dat } \\
\text { *.geo } \\
\text { lightm.dat } \\
\text { xsmorse.bin }\end{array}$ & $\begin{array}{l}\mathrm{m}^{*} \text {.dat } \\
\text { mgams.out } \\
\text { morseh.ppr }\end{array}$ \\
\hline $\begin{array}{l}\text { MEGS } \\
\text { exec.egs }\end{array}$ & $\begin{array}{l}\text { megs.inp } \\
\text { mgams.out } \\
\text { pegs.dat } \\
*^{*} \text { geo }\end{array}$ & $\begin{array}{l}\text { em*.dat } \\
\text { megs.ppr }\end{array}$ \\
\hline $\begin{array}{l}\text { EGSE } \\
\text { exec.egs }\end{array}$ & $\begin{array}{l}\text { egse.inp } \\
(x) \text { e.dat } \\
\text { pegs.dat } \\
* \text { geo }\end{array}$ & $\begin{array}{l}\mathrm{e}^{*} \text { e.dat } \\
\text { egse.ppr }\end{array}$ \\
\hline
\end{tabular}




\section{List of Figures}

Figure 1. Schematic Diagram of CALOR89 code system

Figure 2. Schematic Diagram of CALPREP Program Package

Figure 3. Schematic Diagram of ANALYZ Package

Figure 4. Schematic Diagram of Running CALOR89 Sequentially

Figure 5. Schematic Diagram of Running CALOR89 Concurrently 


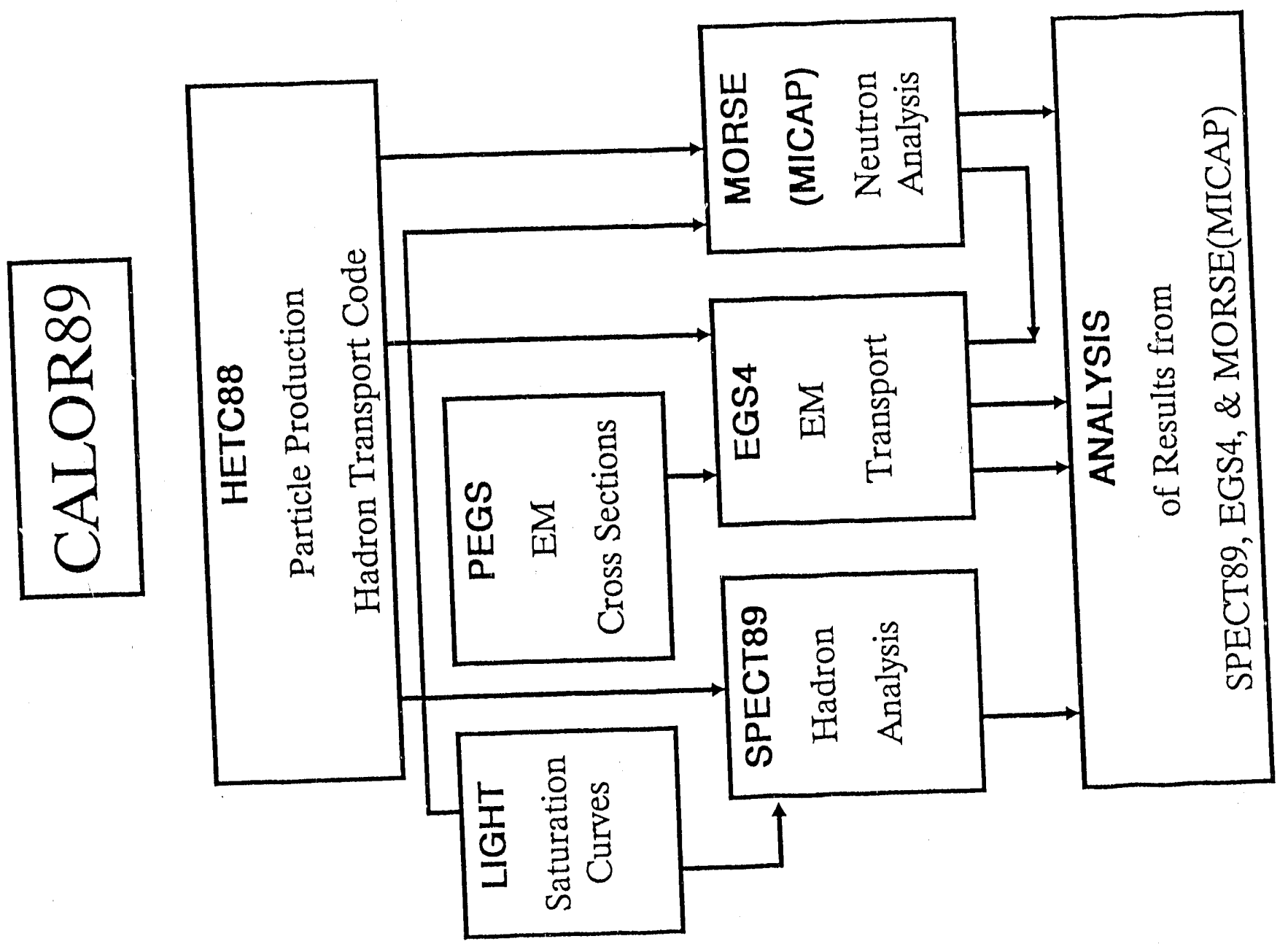

- 


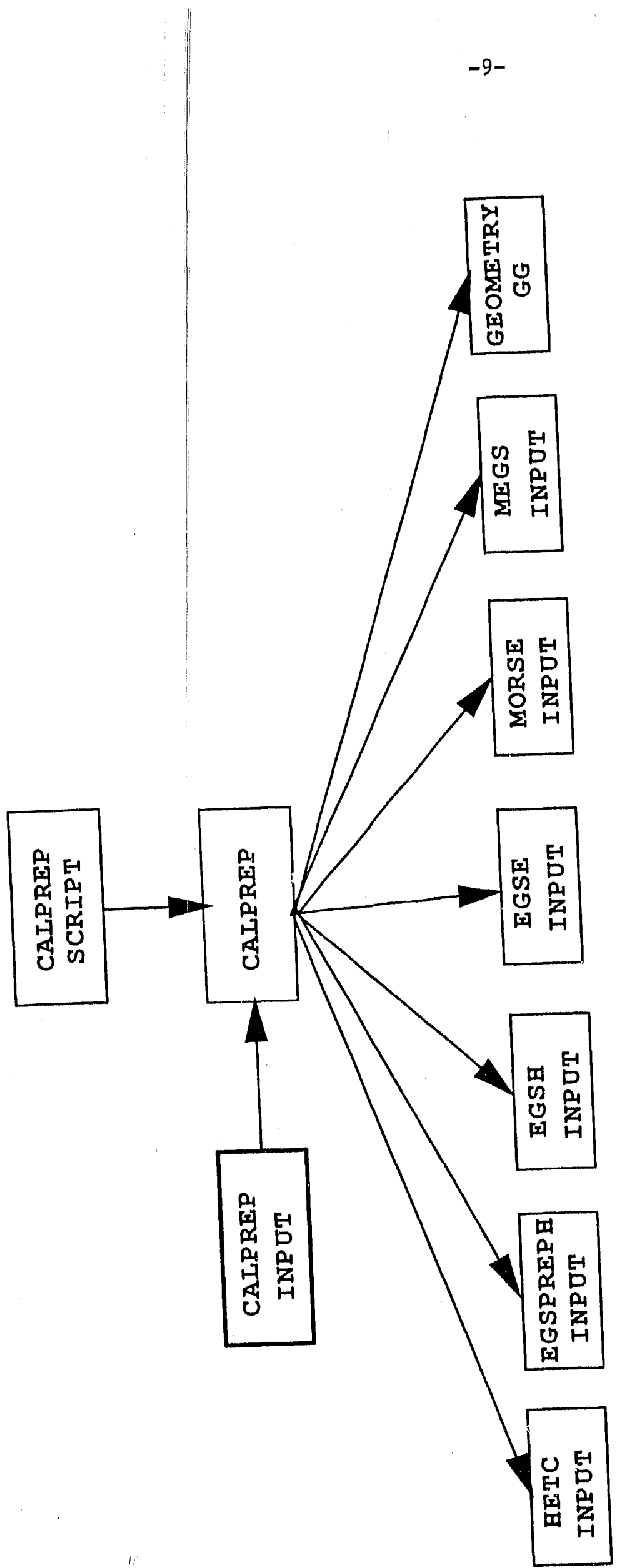

$N$
0
4
5
0
$0-1$
$0=1$ 


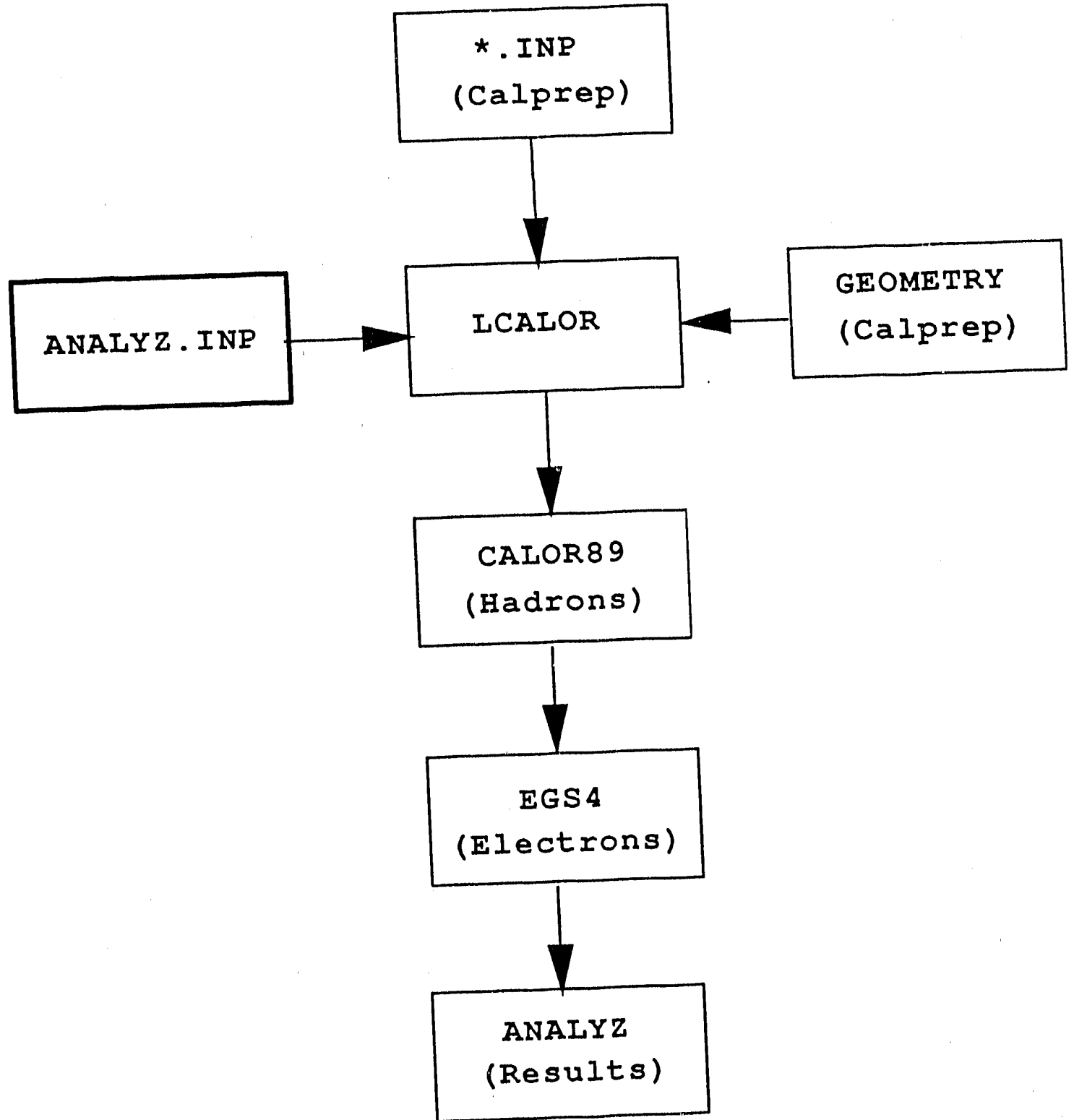

Figure 4 


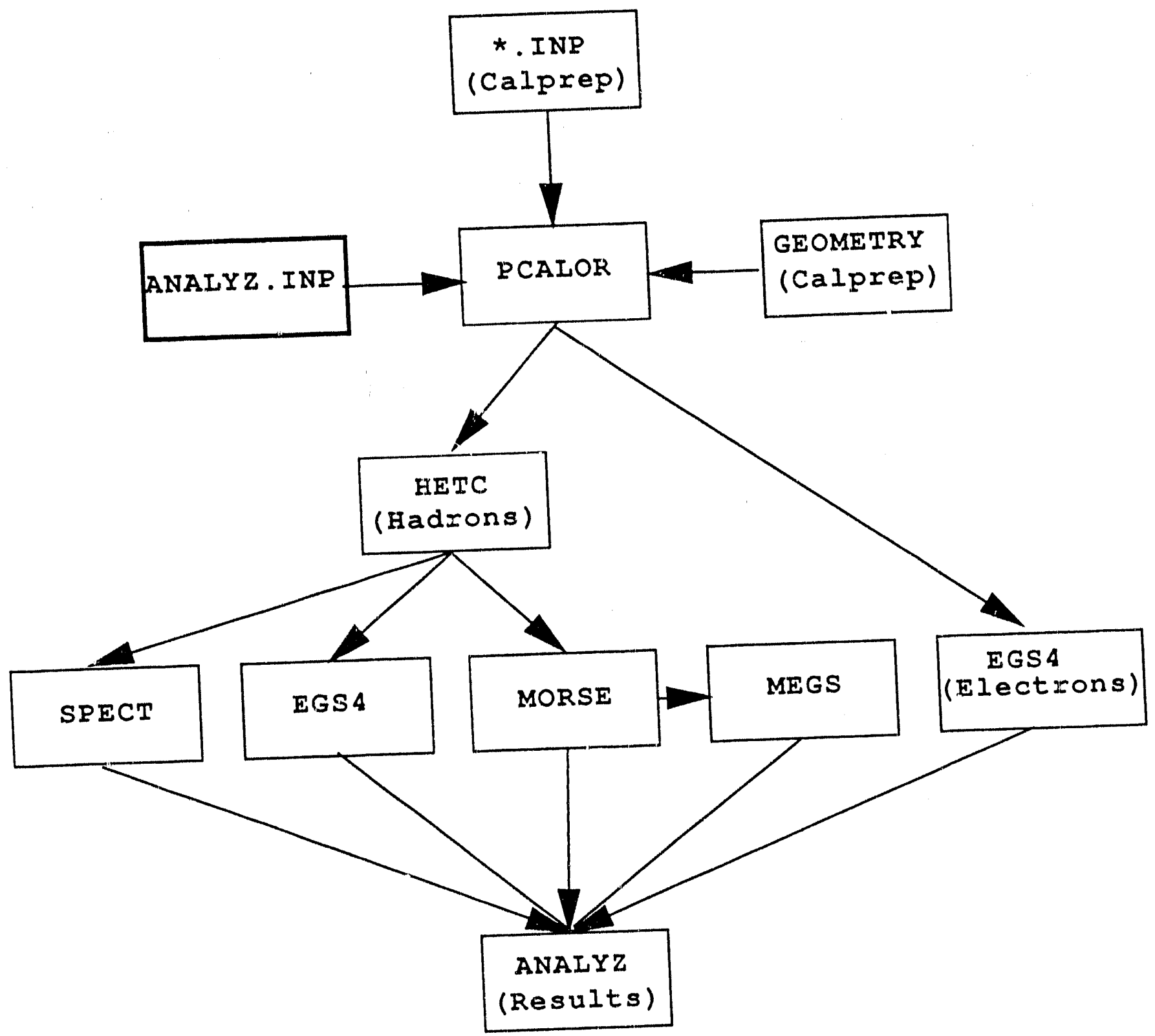

Figure 5 
Data first needed for geometry:

(Material is assumed to begin at $z=0$ and extend to $z>0$ )

First card below is title (up to 80 characters)

- $10 \mathrm{GeV} \quad 3$ Module test configuration'

$\begin{array}{rr}\text { 'PB' } & 12.83 \\ \text { 'PL' } & 2.01\end{array}$

Number of materials (lead,plastic and iron)

'EE' 11.65

Names and $\mathrm{dE} / \mathrm{dx}$

' $\mathrm{PL}$ ' of

$-50.50$.

-50.50 .

3

36

28

17

2

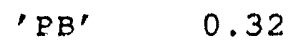

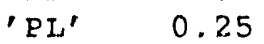

materials (names must agree with PEGS file)

Name of sensitive material

$x \sin , x \max$

ymin, ymax

Number of depth segments (max=5)

2

\# celis in first scgment

\# cella in 2nd segment

\# cells in 3 rd segment

\# materials in lst seg unit cell (max=10)

First segment: first matexial, plate thickness (cm)

2nd material, plate thickness

\# materials in 2nd seg unit cell

' $\mathrm{PB}$ ' 2.10

Second segment: first material, thickness $(\mathrm{cm})$ 2nd material

2

0.25

\# materials in 3 rd seg unit cell

'FE' 5.08

Third segment: first material

$\begin{array}{ll} \\ \text { PL' } & 0.25\end{array}$

2nd material

Data first needed for HETC:

'test.geo" Geometry input file

'test.dat"

HETC binary output file (large)

37591

$1 C 140$.

Random number seed for HETC

Total Energy of incoming particle (MeV)

500

Number of batches (histories)

Data first needed for SORS:

10000 .

15000 .

Kinetic energy (without mass term)

ESCALE (MeV)

4.

0 .

0.01

0 .

0 .

1 .

Particle type $(0=p, 1=n, 2=p i+, 4=p i-, \quad 5=m u+.6=m !-)$

$\times 1$

$y+$ source position (cms)

z 1

1

+ Source direction cosines

non-non

Data first needed for SPECT:

1.

Saturation: $0=1$ inear 1 =nonlinear (run IIGHT for curves)

stest. iat'

spect output file

'/Net/sgi1/sdc2/pkj/spect89/kb131.dat'

13793

Random mumber seed for SPECT

light curves

Data first needed for EGSPREPH

0 0 0 EGS used with HETC, 1=Standalone ESS

$0 \quad 0=$ No Low E gammas, $-1=1$.e. and fission, $1=1$.e. not fisjion

10

13793

'egspreph.out'

35569

\# of source particles printed

Random number seed for EGSPREPH

egsh random number

'/Net/sgil/sdc2/pkj/pegs/pbfeal3.p' pEGS cross section file

'etest.dat' egsh output file

35569 egse random number

'/Net/sgil/sdc3/pkj/calor/egse/10e.dat' electron source file

'etestie.dat'

35569 egse (electrons) output file

'emtest.dat' megs random number megs output file

Data first needed for MORSE

5000

Max \# neutrons handied within MORSE per batch 
105

26

134

4

5

4

$500 \cdot e-9$

13793

, $\begin{array}{rr}46-9 \\ 1 & 13 \\ \text { st. dat }\end{array}$
Number of neutron groups

Number of gamma groups

Cross sertion table length

Number of coefficients for $x$-section

Numbex of basic element cross sections read

Number of mix specifications

Time cut

Random number seed for MORSE

'mtest.dat' sections id?

17
Morse sutput file 


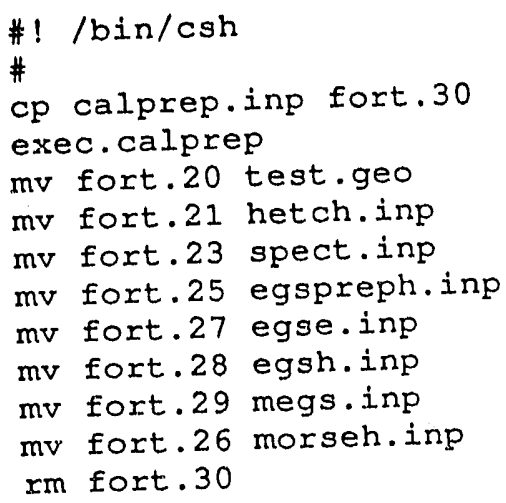


3 module test configuration

Hadron Energy $10 \mathrm{GeV}$

stest.dat

etest.dat

mtest.dat

emtest.dat.

eteste.dat

10.0

card 1 (8i10) number of longitudinal segments in the calorimeter card 2 (8i10) number of cells in each segment

card $3(8 \pm 10.4)$ weight factors by which signal to be enhanced

card 4 (8i10) number of CALOR runs to be analysed

card $5(8 \pm 10)$ maximum hadron/electron energy used for plots

card 6 (a80) general title

card 7 (a80) specific title

The next five cards are the names of the data files for each run given in the order of spect, egs,morse,megs and egse(electrons) output files. For each subsequent runs to be analysed, these file names are given in the same order $(a 80)$.

card 13 ( $8 f 10)$ maximum hadron/electron energy per run 
\#! /bin/csh -f

APPENDIX D

* This script will run the CALOR89 sequence of programs in the foreground.

* Set default of runflag to yes

set runflag = "yes"

\# Check that the number of arguments used is 0 or 1

if (\$\#argv > 1) goto USAGE

\# the following is the set of files that must exist in the current directory. set efiles $=$ (hetch.inp egspreph.inp egsh. inp morseh.inp megs.inp lightm.dat)

\# the following lists the set of files that must exist in the current file

\# and be executable.

set $\mathrm{xfiles}=($ / Net/sgil/sdc3/pkj/hetc/exec.hetc $)$

/Net/sgil/sdc3/pkj/calor/exec.spectl

/Net/sgil/sdc2/pkj/egs/exec.egsprepl

/Net/sgil/sdc3/pkj/calor/exec.egsl

/Net/sgil/sdc3/pkj/calor/exec.morse)

\# Check that all of the efiles exist

foreach flnm (\$efiles[*])

if. $(!-e$ $\$ I n m)$ then

echo The file flnm does not exist in this directory.

echo Either change to the right directory or move \$flnm to this one!

echo

set runflag = "no"

endif

end

\# Check that all of the xfiles exist and have their execute permission on foreach flnm (\$xfiles $[*]$ )

if $(-e$ e $\$ \mathrm{Inm})$ then

if $(!-x$ \$flnm) then

echo The program $\$ f l n m$ is not executable.

echo Use the chmod $u+x$ \$flnm command and make it so!

echo

set runflag = "no"

endif

else

echo The file $\$$ flnm does not exist in this directory.

echo Either change to the right directory or move \$flnm to this one!

echo

set runflag = "no"

endif

end

if (\$runflag != "yes") then

echo CALOR will not execute until the above actions are taken!

else

switch $(\$ 1)$

\# If no arguments, run calor from the top.

case "':

echo

echo starting CALOR from HETC!

date ' $+8 \mathrm{~h}$ \&d, $8 \mathrm{~T}$ '

\# HETC

echo

mv -f hetch.inp hetc.inp

echo starting exechetc.

date ' $+8 \mathrm{~h}$ \&d, $8 \mathrm{~T}$ '

/Net/sgil/sdc2/pkj/hetc/exec.hetc

mv $-f$ hetc.inp hetch.inp

* Start SRECT if arg is SRECT in either upper or lower case 
echo

case $[s S][p P][e E][C C][t T]:$

echo starting exec.spect.

date 'toh $8 d$, $8 \mathrm{~T}$ '

/Net/sgi1/sdc3/pkj/calor/exec.spect

\# Start EgSPREP if arg is EGSP in either upper or lower case t cho case $[e E][g G][s S][p P]:$

mv -f egspreph.inp egsprep.inp

echo starting exec.egsprep.

date 'toh od, $z T$ '

/Net/sgil/sdc2/pkj/egs/exec.egsprep

mv -f egsprep.inp egspreph.inp

$m v$ - $f$ egsprep.ppr egspreph.ppr

\# Start EGS is arg is EGS in either upper or lower case

echo case $[e E][g G][s S]:$

mv -f egsh.inp egs.inp

echo starting exec.egs.

date 'toh $8 \mathrm{~d}$, $8 \mathrm{~T}$ '

/Net/sgil/sdc3/pkj/calor/exec.egs

mv -f egs.inp egsh.inp

$m v-f$ EGS.PPR egsh.PPr

rm -f egspreph.out

\# Start MORSE if arg is MORSE in either upper or lower case

echo

case $[\mathrm{mM}][00][\mathrm{rR}][\mathrm{sS}][\mathrm{eE}]$ :

mv -f morseh.inp morse. inp

echo starting exec.morse.

date 'toh ofd, $8 \mathrm{~T}$ '

/Net/sgil/sdc3/pkj/calor/exec.morse

mv -f morse.ppr morseh.ppr

mv -f morse.inp morseh.inp

\# Start EGS again if arg is MEGS in either upper or lower case echo case [mM] [eE] [gG] [sS]:

mv -f megs.inp egs.inp

echo starting exec.egs again.

date ' $+8 \mathrm{~h}$ \&d, $8 \mathrm{~T}$ '

echo

/Net/sgil/sdc3/pkj/calor/exec.egs

mv -f egs.inp megs. inp

$m v-f$ EGS.PPR megs.ppr

rm - $f$ mgams.out

echo starting exec.egs again.

date ' $+8 \mathrm{~h}$ id, $8 \mathrm{~T}$ '

echo

$m v$-f egse.inp egs.inp

echo

/Net/sgil/sdc3/pkj, alor/exec.egs

mv -f egs.inp egse.inp

echo starting exec.analyz

date ' $+8 \mathrm{~h}$ od, $8 \mathrm{~T}$ '

echo

breaksw

/Net/sgil/sdc3/pkj/calor/exec.analyz

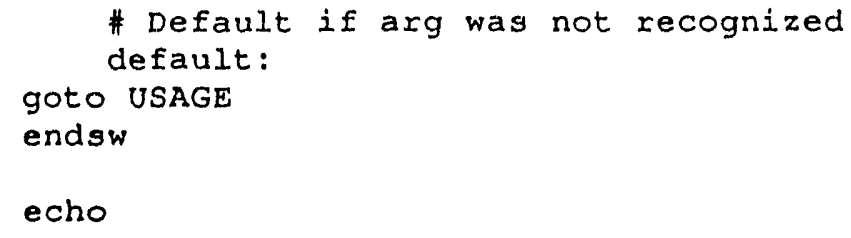


echo CALOR completed!

date ' $+8 \mathrm{~h}$ \&d, $8 \mathrm{~T}$ '

endif

goto END

\section{USAGE :}

echo 'syntax error, USAGE: calor [SRECT or EGSP or EGS or MORSE or MEGS]'

echo 'calor should be called with $\mathrm{ONE}$ of the above arguments or No arguments.'

END : 
\# This gcript will run the CALOR89 sequence of programs in parallel.

unset notify

\# Set default of runflag to yes

set runflag = "yes"

\# Check that the number of arguments used is 0 or 1

if (\$\#argv > 1) goto USAGE

\# the following is the set of files that must exist in the current directory. set efiles $=$ (hetch.inp egspreph.inp egsh.inp morseh.inp megs.inp lightm.dat)

\# the following lists the set of files that must exist in the current file

\# and be executable.

set $x$ files $=(/ \mathrm{Net} / \mathrm{sgil} / \mathrm{sdc} 3 / \mathrm{pkj} /$ hetc/exec.hetcl

/Net/sgil/sdc3/pkj/calor/exec.spect।

/Net/sgi1/sdc2/pkj/egs/exec.egsprepl

/ Net/sgi1/sdc3/pkj/calor/exec.egs/

/Net/sgi1/sdc3/pkj/calor/exec.morse)

\# Check that all of the efiles exist

foreach flnm (Sefiles[*])

if (! -e $\$ f I n m)$ then

echo The file $\$ f l n m$ does not exist in this directory.

echo Either change to the right directory or move \$flnm to this one!

echo

set runflag $=$ "no"

endif

end

\# Check that all of the xfiles exist and have their exscute permission on foreach flnm (\$xfiles[*])

if $(-\mathrm{e} \$ f l n m)$ then

if $(!-x$ \$flnm) then

echo The program $\$ f l n m$ is not executable.

echo Use the chmod $u+x$ fflnm command and make it so!

echo

set runflag $=$ "no"

endif

else

echo The file $\$ f l n m$ does not exist in this directory.

echo Either change to the right directory or move \$flnm to this one!

echo

set runflag $=$ "no"

endif

end

if (\$runflag $!=$ "yes") then

echo CALOR will not execute until the above actions are taken!

else

switch (\$1)

\# If no arguments, run calor from the top.

case' ' :

echo

echo starting CALOR from HETC!

date 'toh $z d, \quad z T$ '

\# HETC

echo

mv -f hetch.inp hetc.inp

echo starting exechetc.

date ' $+8 \mathrm{~h}$ \&d, $8 \mathrm{~T}$ '

/Net/sgil/sdc3/pkj/hetc/exec.hetc 
mo

-f hetc.inp hetch.inp

\# Start SPECT if arg is anything. default :

\# Make directory to run megs in.

mkdir ./temp.megs

$m v$-f megs.inp./temp.megs/egs.inp

$\mathrm{cp} / \mathrm{Net} / \mathrm{sgi1/sdc3/pkj/cal.or/exec.egs//temp.megs/exec.megs}$

\# Make directory to run egse(electrons) in.

mkdir . /temp.egse

mv -f egse.inp./temp.egse/egs.inp

$\mathrm{cp} / \mathrm{Net} / \mathrm{sgil} / \mathrm{sdc} 3 / \mathrm{pkj} / \mathrm{cal}$ or/exec.egs ./temp.egse/exec.egse

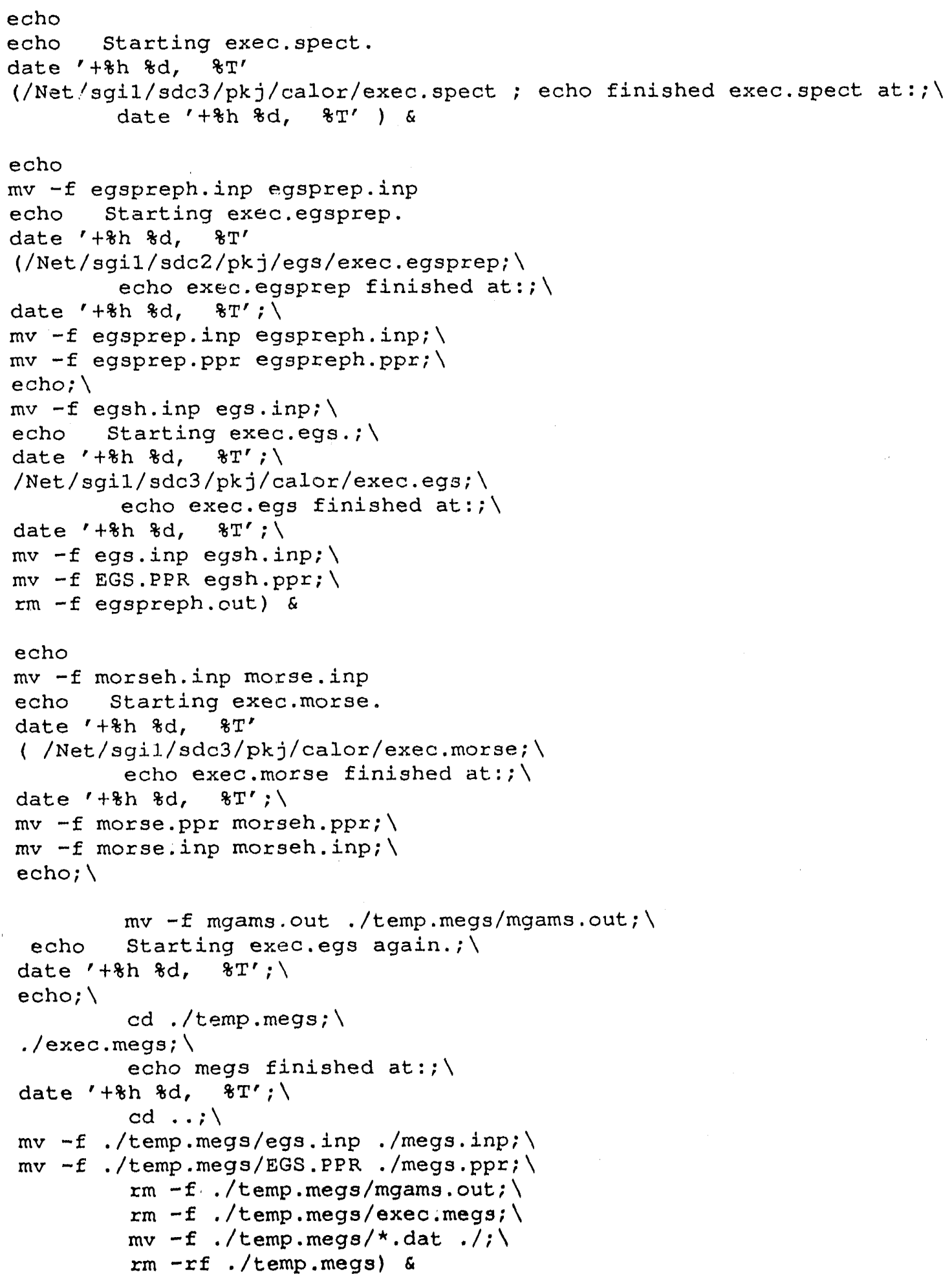




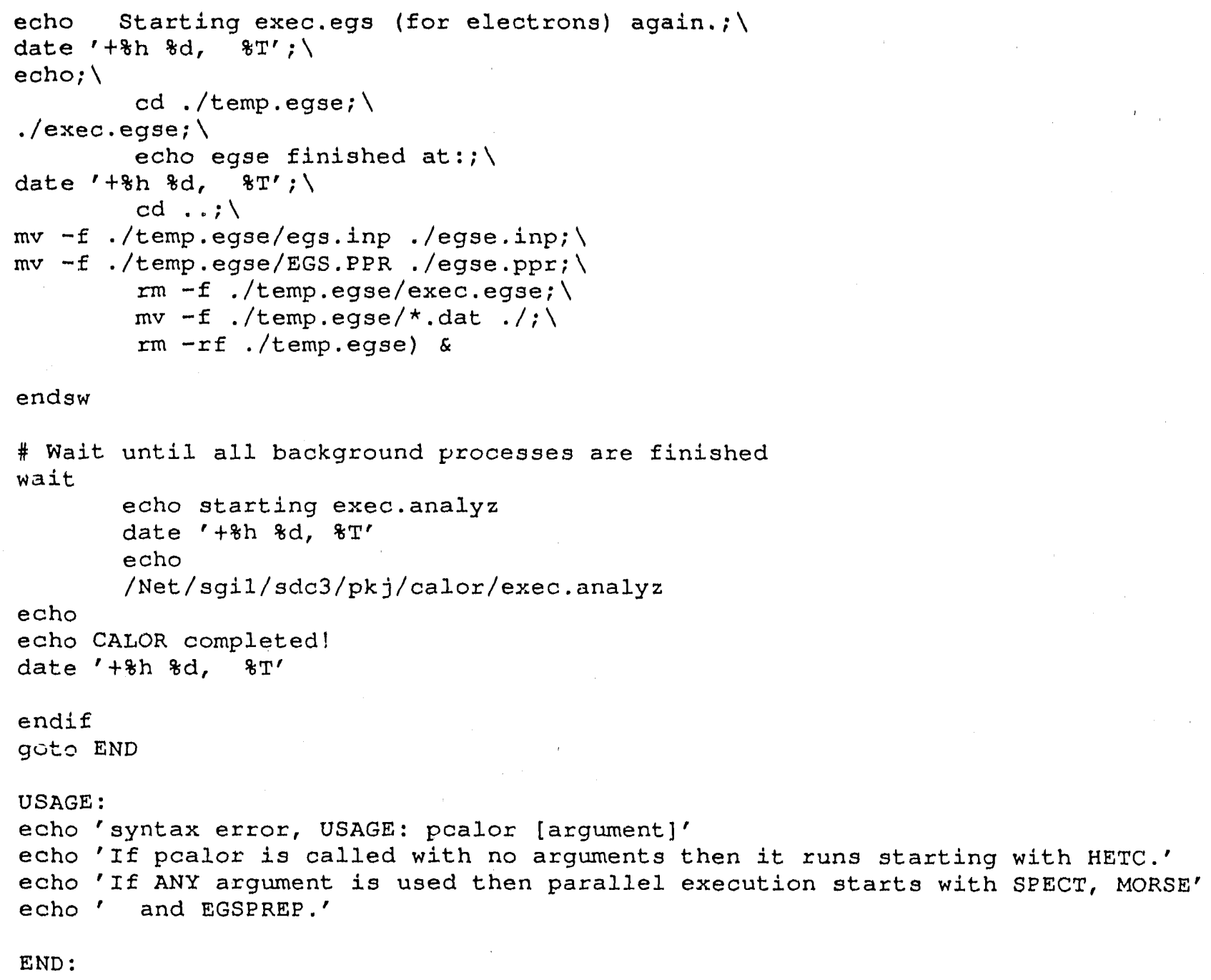

END : 


\section{2}
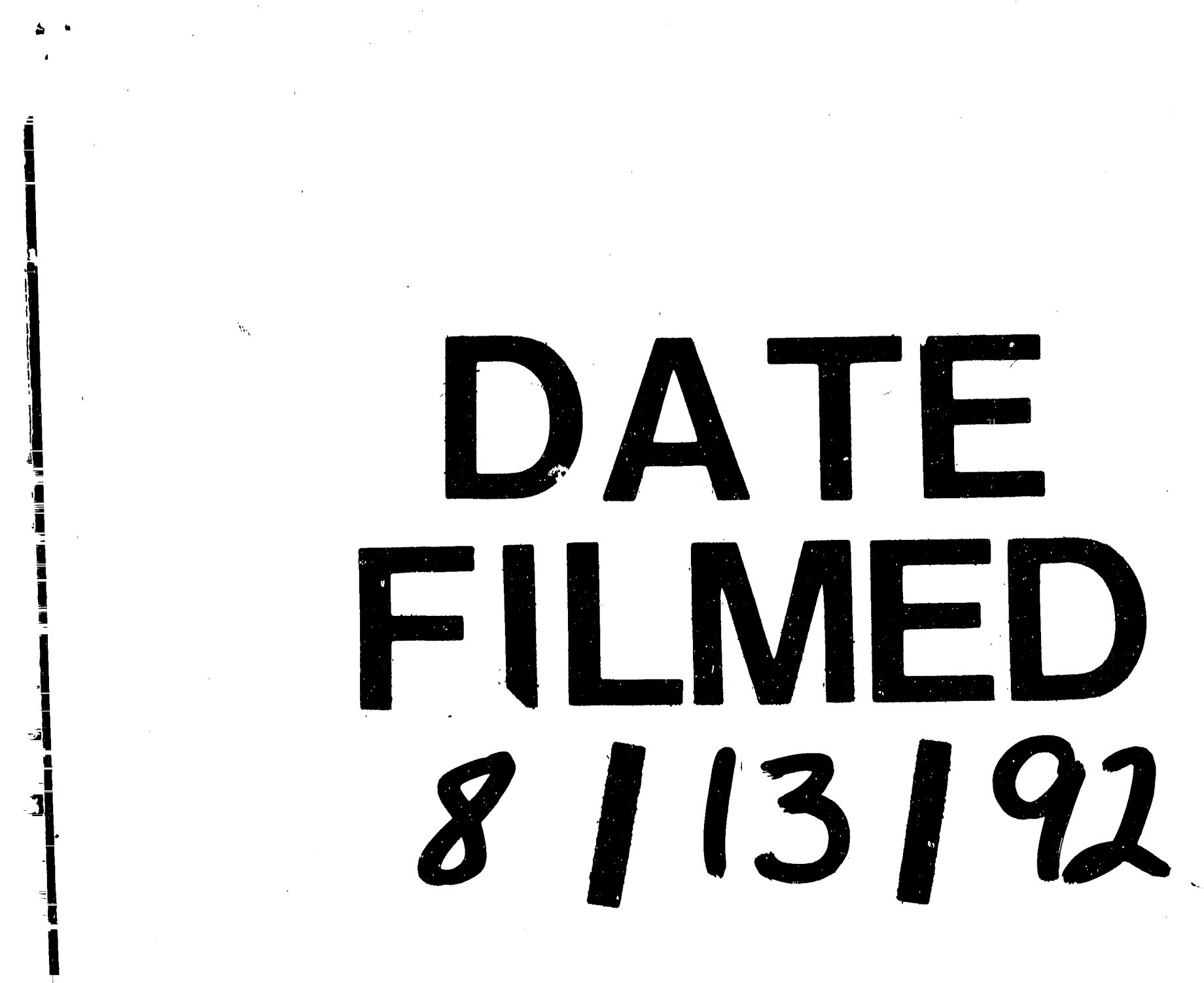
